\title{
A Unified Simulation Approach for the Fast Outage Capacity Evaluation over Generalized Fading Channels
}

\author{
Nadhir Ben Rached, Abla Kammoun, Mohamed-Slim Alouini, and Raul Tempone \\ King Abdullah University of Science and Technology (KAUST) \\ \{nadhir.benrached, abla.kammoun, slim.alouini, raul.tempone\}@kaust.edu.sa
}

\begin{abstract}
The outage capacity (OC) is among the most important performance metrics of communication systems over fading channels. The evaluation of the $\mathrm{OC}$, when Equal Gain Combining (EGC) or Maximum Ratio Combining (MRC) diversity techniques are employed, boils down to computing the Cumulative Distribution Function (CDF) of the sum of channel envelopes (equivalently amplitudes) for EGC or channel gain (equivalently squared enveloped/amplitudes) for MRC. Closed-form expressions of the CDF of the sum of many generalized fading variates are generally unknown and constitute open problems. In this paper, we develop a unified hazard rate twisting Importance Sampling (IS) based approach to efficiently estimate the CDF of the sum of independent arbitrary variates. The proposed IS estimator is shown to achieve an asymptotic optimality criterion, which clearly guarantees its efficiency. Some selected simulation results are also shown to illustrate the substantial computational gain achieved by the proposed IS scheme over crude Monte-Carlo simulations.
\end{abstract}

Index Terms-Outage capacity, fading channels, hazard rate twisting, Importance Sampling, asymptotic optimality.

\section{INTRODUCTION}

Diversity techniques have been extensively investigated due to their efficiency in combating fading and hence improving the performance of wireless communication systems [1]. Of particular interest are the Maximum Ratio Combining (MRC) and Equal Gain Combining (EGC) diversity receivers operating over fading channels. Understanding the performance of these schemes is of valuable practical interest. Among the commonly used performance metrics, we distinguish the outage capacity which is defined as the probability that the instantaneous capacity falls below a certain threshold. When MRC or EGC diversity technique are employed, the problem of evaluating the outage capacity is equivalent to computing the Cumulative Distribution Function (CDF) of the sum of the fading envelopes for EGC or the channel gains for MRC. Generally, the CDF of the sum of many challenging distributions is unknown in closed-form. A non exhaustive list of such interesting problems includes the sum of Lognormal Random Variables (RVs), widely used in the context of cellular communication systems [2], the sum of GammaGamma RVs well-suited to model fading model in free space optical links [3], and that of Weibull RVs known for its good fit to experimental fading channel measurements of indoor and outdoor environments [4]. To tackle this issue, a considerable research effort has been carried out to provide approximations of the CDF of the sum of given RVs. These approximations can be mainly classified into four categories. The first category includes approaches that approximate the considered sum of RVs by another RV. We cite for instance the work in [5] where the sum of Log-normal RVs was approximated by another Log-normal. In the same vein, the $\alpha-\mu$ PDF envelop was used to approximate the sum of Weibull [6], Nakagami [7], and Gamma-Gamma [8] variates. The second class of approximations is based on providing closed-form approximations of the CDF of a set of sum distributions including the Log-normal [9] and the Rayleigh [10] RVs. Although the approximations in these two categories were shown to exhibit a good level of accuracy, there is no guarantee that they remain accurate for all range of probabilities and for arbitrary number of summands. For instance, a clear discrepancy is observed if the sum of Log-normal RVs is approximated by another Log-normal variate [11]. Furthermore, the parameters of these closedform expressions are often determined by solving a nonlinear least squares problem, which requires the computation of the empirical CDF of the sum distributions [9], and thus might lead to high computational complexities. To overcome this issue, techniques based on efficient numerical integration have been proposed. These latter include for instance an approximation method based on a Smolyak's algorithm [12] which has been developed to deal with the distribution of the sum of Log-normal RVs. Finally, a fourth class of techniques based on the derivation of infinite sum expansion have been also provided. We cite, for example, the work in [13] where a simple infinite Gamma series expansions has been developed to approximate the CDF of a sum of squared RVs, namely the Nakagami and the Rice fading models . The previously cited approximation techniques share the common denominator of being non generic, that is, particularly applicable to a given distribution (or a narrow class of distributions). To the best of our knowledge, a unified approach to approximate the CDF of a sum of arbitrary RVs does not exist. This principally constitute the major motivation behind this work. In particular, we aim in this paper to develop a unified, yet efficient, Importance Sampling (IS) based framework to estimate the $\mathrm{CDF}$ of a sum of arbitrary independent variates. The adopted methodology consists in performing necessary problem adjustments to the hazard rate twisting IS approach developed originally in [14] and recently revisited in [15], in order to adapt it to the problem of interest. The main result is that the proposed IS estimator possesses an asymptotic optimality criterion for sums involving independent arbitrary variates. Such a generalized result achieves one of the most sought-after requirement in the context of variance reduction algorithms 
and thus represents a relevant novelty, mainly because, in this field, optimality results were often proved for particular classes of distributions. The rest of the paper is organized as follows. In Section II, the problem setting of our work is described. The methodology of our approach is presented in Section III. In section IV, the main theorem proving the asymptotic optimality property is stated. Prior to concluding, selected simulation results are presented in order to illustrate the substantial computational gain achieved by the proposed IS approach over crude Monte-Carlo simulations.

\section{Problem Setting}

The instantaneous Signal-to-Noise Ratio (SNR) at the output of $N$-branch MRC/EGC receiver can be written as [16]

$$
\gamma_{\text {end }}=\frac{E_{s}}{N_{0} \sqrt{N^{1-p+q}}}\left(\sum_{i=1}^{N} R_{i}^{p}\right)^{q}
$$

where $N$ is the number of diversity branches, $E_{s} / N_{0}$ is the SNR per symbol at the transmitter, and $R_{i}, i=1,2, \ldots, N$, is the $i^{t h}$ fading envelope. The parameter $p$ and $q$ in (1) are as follows:

$$
(p, q)= \begin{cases}(1,2), & \text { EGC } \\ (2,1), & \text { MRC }\end{cases}
$$

The OC is among the most important performance metrics of communication systems operating over fading channels. This metric is defined as the probability that the instantaneous capacity $C_{\gamma_{\text {end }}}=\log _{2}\left(1+\gamma_{\text {end }}\right)$ falls below a certain threshold $C_{t h}$, that is

$$
C_{\text {out }}=P\left(0 \leq C_{\gamma_{\text {end }}}<C_{t h}\right) .
$$

From (1), it can be seen that the outage capacity $C_{\text {out }}$ writes as:

$$
C_{\text {out }}=P\left(\sum_{i=1}^{N} R_{i}^{p}<\left(\frac{N_{0} \sqrt{N^{1-p+q}}}{E_{s}}\left(2^{\frac{C_{t h}}{W}}-1\right)\right)^{\frac{1}{q}}\right) .
$$

Hence, computing the outage capacity with MRC/EGC diversity techniques is equivalent to determining the $\mathrm{CDF}$ of the sum of the fading envelopes (EGC) or the channel gains (MRC). In a higher level of abstraction, our goal is then to develop an efficient algorithm of the quantity

$$
\alpha=P\left(S_{N}=\sum_{i=1}^{N} X_{i}<\gamma_{t h}\right),
$$

for a given threshold $\gamma_{t h}$, where $X_{1}, X_{2}, \ldots, X_{N}$ is a sequence of independent and not necessarily identically distributed positive RVs with Probability Density Function (PDF) $f_{X_{i}}(\cdot)$, $i=1,2, \ldots, N$. In typical wireless communication systems, the OC is relatively small and thus a special focus will be given to estimating the probability of the rare event $\left\{\sum_{i=1}^{N} X_{i}<\gamma_{t h}\right\}$ when $\gamma_{t h}$ is sufficiently small. The standard approach based on $\mathrm{MC}$ simulations leads to estimate $\alpha$ by the following crude MC estimator:

$$
\hat{\alpha}_{M C}=\frac{1}{M} \sum_{i=1}^{M} \mathbf{1}_{\left(S_{N}\left(\omega_{i}\right)<\gamma_{t h}\right)},
$$

where $M$ is the number of simulation runs and $\mathbf{1}_{(\cdot)}$ defines the indicator function. In the setting of rare events simulations, i.e. events with very small probabilities, crude MC simulations are known to be computationally expensive. IS is a variance reduction technique that can overcome the failure of crude $\mathrm{MC}$ simulations and considerably reduce the computational work [17]. IS techniques are based on performing a change of the probability measure, in that sampling is performed according to a new distribution instead of the original one. The aim is mainly to emphasize sampling of important values, i.e. values which have more impact on the parameter of interest $\alpha$ than others. More precisely, the change of the sampling distribution is performed in a way to increase the frequency of realizations belonging to the rare event $\left\{S_{N}<\gamma_{t h}\right\}$, when $\gamma_{t h}$ is small enough. This allows to reach an interesting amount of variance reduction compared to crude MC simulations.

\section{Methodology}

\section{A. Hazard Rate Twisting Technique}

To the best of our knowledge, the exponential twisting approach, derived from the large deviation theory, is considered as the most interesting IS change of measure [18]. However, this approach presents the drawback of requiring the moment generating function (MGF) of each RV in the sum to be known in closed-form. Such a requirement does not hold for many generalized fading variates. We can cite for sake of illustration, the Log-normal, the Weibull and the Nakagami RVs. In the present work, we propose an alternative IS algorithm that not only avoids using the MGF but also is applicable to arbitrary sums of independent RVs. Our methodology is based on the well known hazard rate twisting technique introduced in [14]. This approach was originally developed to efficiently estimate the probability of the rare set $\left\{S_{N}>\gamma_{t h}\right\}$, where $\gamma_{t h}$ is sufficiently large and the components of $S_{N}$ are independent and identically distributed with subexponential right-tail decay. The method in [14] was extended in [15] to problems involving sums of independent arbitrary RVs prior to being further extended in [19]. These methods cannot be directly used to address the considered problem of the present work, since the rare set of interest here is $\left\{S_{N}<\gamma_{t h}\right\}$, where $\gamma_{t h}$ is sufficiently small. As far as the authors know, the use of hazard rate twisting technique to estimate the probability of such events has not previously been addressed, which makes it the major contribution of our work.

\section{B. Proposed Approach}

To facilitate the understanding of the proposed method, we shall first recall the principle of the hazard rate twisting [15]. This is an important step that will help the reader understand how we adapt the technique of [15] to the considered problem. In [15], the probability of interest, i.e. the probability of the event $\left\{S_{N}>\gamma_{t h}\right\}$ for large $\gamma_{t h}$, is re-expressed as follows

$$
P\left(S_{N}=\sum_{i=1}^{N} X_{i}>\gamma_{t h}\right)=\mathbb{E}_{\theta}\left[\mathbf{1}_{\left(S_{N}>\gamma_{t h}\right)} \prod_{i=1}^{N} \frac{f_{X_{i}}\left(X_{i}\right)}{f_{X_{i}, \theta}\left(X_{i}\right)}\right],
$$


where the expectation $\mathbb{E}_{\theta}[\cdot]$ is with respect to new PDFs $f_{X_{i}, \theta}(\cdot)$ obtained after twisting the hazard rate of $X_{i}, i=$ $1,2, \ldots, N$, by a quantity $0 \leq \theta<1$, that is

$$
f_{X_{i}, \theta}(x)=(1-\theta) \lambda_{X_{i}}(x) \exp \left(-(1-\theta) \Lambda_{X_{i}}(x)\right), \quad x>0,
$$

with $\lambda_{X_{i}}(x)=\frac{f_{X_{i}}(x)}{1-F_{X_{i}}(x)}$ and $\Lambda_{X_{i}}(x)=-\log \left(1-F_{X_{i}}(x)\right)$ denote respectively the hazard rate and the hazard function. A key characteristic of this approach [15] is that it puts much more emphasis to samples belonging to the rare set $\left\{S_{N}>\right.$ $\left.\gamma_{t h}\right\}$, for a sufficiently large $\gamma_{t h}$, at the expense of samples belonging to the rare set $\left\{S_{N}<\gamma_{t h}\right\}$ for small values of $\gamma_{t h}$. This interesting feature is indeed obtained since the twisted sum distribution is more heavier to the right than the original one. To illustrate this statement, we plot in Fig. 1.a the twisted and untwisted PDFs of $S_{N}$. We can easily observe that samples belonging to the set $\left\{S_{N}>\gamma_{t h}\right\}$, for sufficiently large $\gamma_{t h}$, are more likely to occur under the twisted PDF than under the untwisted one. Since the opposite occurs for the set $\left\{S_{N}<\right.$ $\left.\gamma_{t h}\right\}$ with $\gamma_{t h}$ sufficiently small, a direct application of the approach in [15] to the present problem will actually lead to worse computational efficiencies compared to the crude Monte Carlo simulations. An adjustment is therefore required. To this

(a)
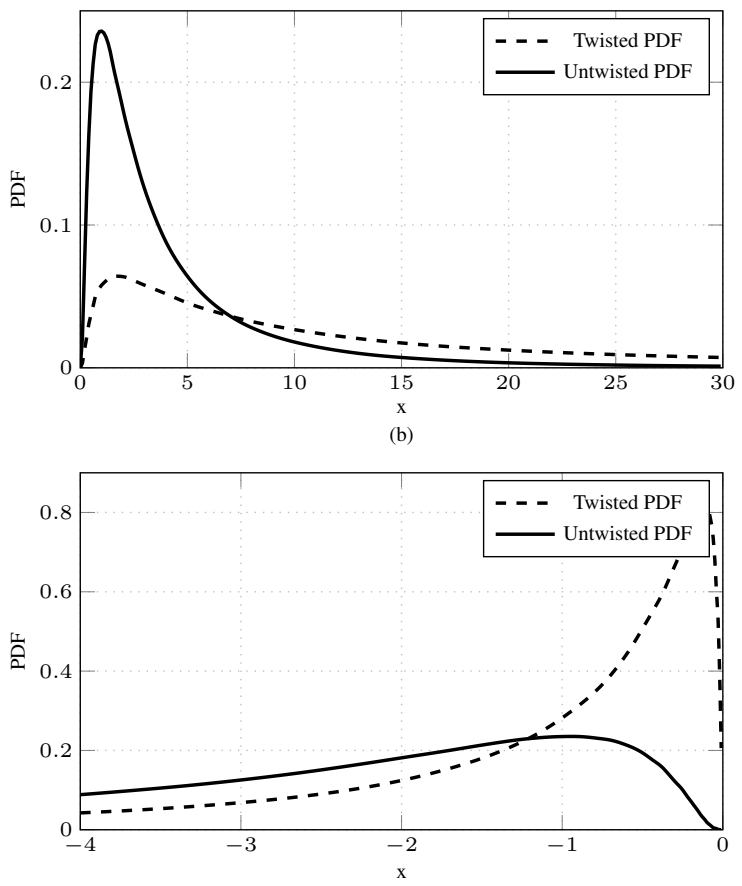

Fig. 1. Twisted and original PDFs: a PDF of $S_{2}$ with $X_{1}$ and $X_{2}$ are i.i.d Log-normal RVs with $\mu=0 \mathrm{~dB}, \sigma=6 \mathrm{~dB}$ and $\theta=0.6$, (b) PDF of $-S_{2}$ with $\theta=0.6$.

end, we start by rewriting the probability of interest $\alpha$ as:

$$
\alpha=P\left(S_{N}=\sum_{i=1}^{N} X_{i}<\gamma_{t h}\right)=P\left(\sum_{i=1}^{N} Y_{i}>-\gamma_{t h}\right)
$$

where $Y_{i}=-X_{i}, i=1,2, \ldots, N$. Instead of applying the hazard rate twisting technique to $X_{i}, i=1,2, \ldots, N$ as in [15], we propose to apply it to $Y_{i}, i=1,2, \ldots, N$. More formally, the following change of probability measure is performed:

$$
\alpha=\mathbb{E}_{\theta}\left[\mathbf{1}_{\left(S_{N}<\gamma_{t h}\right)} L\left(Y_{1}, Y_{2}, \ldots, Y_{N}\right)\right],
$$

where $\mathbb{E}_{\theta}[\cdot]$ is now taken under the PDFs $f_{Y_{i}, \theta}(\cdot)$ which, in the same way as in (8), write as:

$$
f_{Y_{i}, \theta}(y)=(1-\theta) \lambda_{Y_{i}}(y) \exp \left(-(1-\theta) \Lambda_{Y_{i}}(y)\right), \quad y<0,
$$

where $\lambda_{Y_{i}}(y)=\frac{f_{X_{i}}(-y)}{F_{X_{i}}(-y)}$ and $\Lambda_{Y_{i}}(y)=-\log \left(F_{X_{i}}(-y)\right)$. The quantity $L\left(Y_{1}, Y_{2}, \ldots, Y_{N}\right)=\prod_{i=1}^{N} \frac{f_{Y_{i}}\left(Y_{i}\right)}{f_{Y_{i}, \theta}\left(Y_{i}\right)}$ is the likelihood ratio given, according to (11), by:

$$
L\left(Y_{1}, Y_{2}, \ldots, Y_{N}\right)=\frac{1}{(1-\theta)^{N}} \exp \left(-\theta \sum_{i=1}^{N} \Lambda_{Y_{i}}\left(Y_{i}\right)\right) .
$$

Finally, the IS estimator is

$$
\hat{\alpha}_{I S}=\frac{1}{M} \sum_{i=1}^{M} \mathbf{1}_{\left(S_{N}\left(\omega_{i}\right)<\gamma_{t h}\right)} L\left(Y_{1}\left(\omega_{i}\right), Y_{2}\left(\omega_{i}\right), \ldots, Y_{N}\left(\omega_{i}\right)\right) .
$$

The rationale behind this idea is again based on the major feature of the hazard rate twisting technique of leading to heavier right-tail distributions than the original one. Thus, applying this technique to $Y_{i}, i=1,2, \ldots, N$ will certainly favor the sampling of realizations in a neighborhood of 0 (since $Y_{i}, i=1,2, \ldots, N$, are negative), that is realizations belonging to the set $\left\{S_{N}<\gamma_{t h}\right\}$ for a sufficiently small $\gamma_{t h}$. To validate this statement we plot in Fig. 1.b the twisted and untwisted PDFs of $-S_{N}$. We clearly observe that the twisted PDF is more concentrated in the neighborhood of 0 than the original one.

\section{Optimal Minmax Twisting Parameter}

The selection of $\theta$ is performed by following the steps of the minmax approach [15] applied to our adjusted problem. The first step consists in providing an upper bound of the second moment of $T_{\gamma_{t h}}$ defined as:

$$
T_{\gamma_{t h}}=\mathbf{1}_{\left(S_{N}<\gamma_{t h}\right)} L\left(Y_{1}, Y_{2}, \ldots, Y_{N}\right) .
$$

This can be achieved by solving the following minimization problem $(P)$ :

$$
\begin{aligned}
\min _{Y_{1}, \ldots, Y_{N}} & \sum_{i=1}^{N} \Lambda_{Y_{i}}\left(Y_{i}\right) \\
\text { Subject to } & \sum_{i=1}^{N} Y_{i}>-\gamma_{t h}, Y_{i}<0, \quad i=1, \ldots, N .
\end{aligned}
$$

Let $Y_{1}^{*}, Y_{2}^{*}, \ldots, Y_{N}^{*}$ denote the solution of (P). From (12), the second moment of $T_{\gamma_{t h}}$ is bounded by

$$
\mathbb{E}_{\theta}\left[T_{\gamma_{t h}}^{2}\right] \leq \frac{1}{(1-\theta)^{2 N}} \exp \left(-2 \theta \sum_{i=1}^{N} \Lambda_{Y_{i}}\left(Y_{i}^{*}\right)\right) .
$$

The second step in the minmax approach is to minimize the previous upper-bound with respect to $\theta$. After simple computation, the optimal minmax twisting parameter is:

$$
\theta^{*}=1-\frac{N}{\sum_{i=1}^{N} \Lambda_{Y_{i}}\left(Y_{i}^{*}\right)} .
$$


In practice, $(P)$ is solved through numerical optimization methods. However, when the CDF $F_{X_{i}}(\cdot), i=1,2, \ldots, N$, is Log-concave, $(P)$ becomes a convex problem and thus convex optimization algorithms can be employed. Examples of distributions with Log-concave CDF include the Log-normal, the Weibull, and the Gamma distributions [20].

\section{AsYMPtotic OPTIMALITY}

Asymptotic optimality is among the commonly used criteria that account for the efficiency of IS schemes. It was employed for instance in [14] and [15]. From the non-negativity of the variance of $T_{\gamma_{t h}}$, we have for each $\theta$ :

$$
\frac{\log \left(\mathbb{E}_{\theta}\left[T_{\gamma_{t h}}^{2}\right]\right)}{\log (\alpha)} \leq 2
$$

We say that $\alpha$ is asymptotically optimally estimated if the previous inequality holds with equality as $\gamma_{t h}$ goes to zero, that is

$$
\lim _{\gamma_{t h} \rightarrow 0} \frac{\log \left(\mathbb{E}_{\theta}\left[T_{\gamma_{t h}}^{2}\right]\right)}{\log (\alpha)}=2 .
$$

It is important to note that the crude MC simulation fails to optimally estimate $\alpha$ since the limit in (18) is equal to 1 .

Theorem 1. For any sum of independent positive RVs, the asymptotic optimality criterion (18) holds with $\theta=\theta^{*}$ given in (16).

Proof: The proof is performed following the same steps as the proof of Theorem 1 in [15] with tending $\gamma_{t h} \rightarrow 0$ instead of $\gamma_{t h} \rightarrow+\infty$.

\section{Simulation Results}

In this section, we provide some simulation results in order to show the efficiency of the proposed IS scheme over crude MC simulations. We estimate the outage capacity for two popular scenarios, namely, the MRC receiver under Lognormal fading, and the EGC receiver under Nakagami fading. The amount of variance reduction is the performance metric which is used to compare the crude MC simulation with the proposed IS approach. This metric is defined by:

$$
k=\frac{\alpha(1-\alpha)}{\operatorname{var}_{\theta^{*}}\left[T_{\gamma_{t h}}\right]},
$$

where $k$ measures the gain achieved by the proposed IS algorithm over crude MC simulations in terms of the number of simulation runs that is necessary to meet a fixed accuracy requirement.

\section{A. Log-normal Fading with MRC Diversity Reception}

In this subsection, we evaluate the OC of MRC diversity technique operating over Log-normal fading variates, that is the CDF of the sum of squared Log-normal RVs (4). The PDF of the fading envelope $R_{i}, i=1,2, \ldots, N$, is

$$
f_{R_{i}}(r)=\frac{1}{\sqrt{2 \pi} r \sigma_{i}} \exp \left(-\frac{\left(\log (r)-\mu_{i}\right)^{2}}{2 \sigma_{i}^{2}}\right), r>0,
$$

where $\mu_{i}$ and $\sigma_{i}, i=1,2, \ldots, N$, are the mean and the standard deviation of the associated Gaussian distribution. It is worth

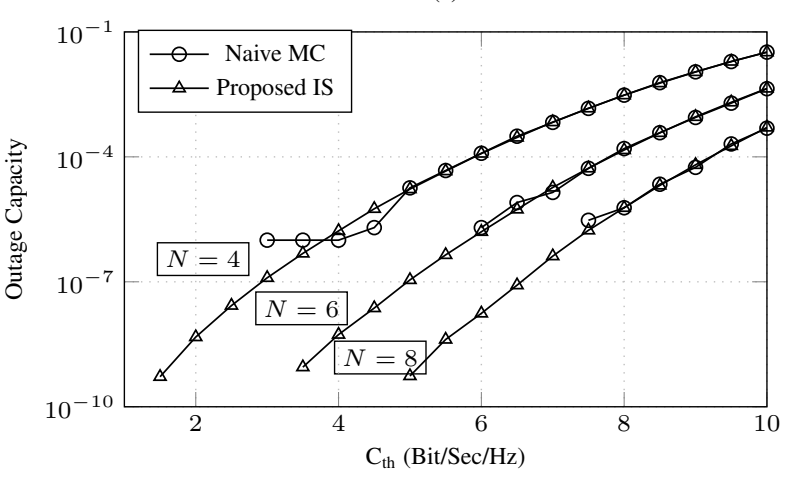

(b)

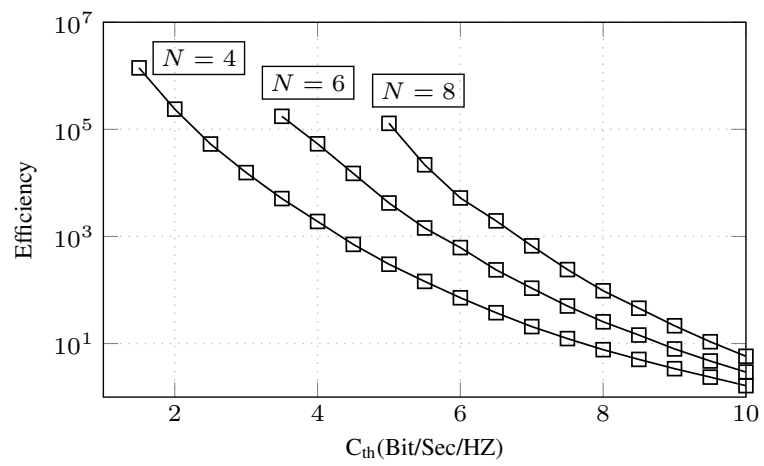

Fig. 2. (a) Outage capacity of $N$-branch $\mathrm{MRC}$ diversity receivers over independent Log-normal fading variates with $\mu_{i}=10 \mathrm{~dB} i=1,2, \ldots, 8$. $N=4: \sigma=\{6,6,6,6\}$ dB. $N=6: \sigma=\{6,6,6,6,8,8\}$ dB $. N=8$ : $\sigma=\{6,6,6,6,8,8,8,8\} \mathrm{dB}$. (b) Efficiency $k$ with same parameters. Number of simulation runs: $M=10^{6}$.

noting that $R_{i}^{2}$ is again a Log-normal RV with parameters $2 \mu_{i}$ and $2 \sigma_{i}, i=1,2, \ldots, N$. In Fig. 2.a the outage capacity of $N$ branch MRC diversity receivers over independent Log-normal fading variates is estimated using both crude MC simulations and the proposed hazard rate twisting IS approach where the twisting parameter is given by (16). The number of simulation runs used in both techniques is $M=10^{6}$. From this figure, the failure of the crude MC simulation is obvious. In fact, for each value of $N$, a clear oscillatory behavior can be noted for small values of the OC. Furthermore, as the threshold $C_{t h}$ decreases, the crude $\mathrm{MC}$ method fails to generate important samples, i.e. realizations that falls below the given threshold, and hence estimates the probability of interest by zero. Thus, much more number of simulation runs are needed to overcome this failure. From the same figure, we easily observe that our proposed IS approach presents a smoother curve, and thus, with the same number of simulation runs, our IS technique is much more accurate than the crude MC simulation. To measure the gain in terms of simulation runs that is achieved by our proposed IS method as compared to crude MC simulations, we plot in Fig. 2.b the amount of variance reduction $k$ defined in (19) using the same parameters as in Fig. 2.a. From this figure, a substantial amount of variance reduction can be clearly noticed. This reduction becomes rapidly much more significant as we decrease the threshold $C_{t h}$, i.e. small values of the OC. For instance, from Fig. 2.b, when $N=4$ and $C_{t h}=1.5$ $\mathrm{Bit} / \mathrm{Sec} / \mathrm{Hz}$, the crude $\mathrm{MC}$ simulations requires approximately $10^{6} \times M$ simulations runs to retrieve the accuracy of the IS approach with $M$ simulation runs. 


\section{B. Nakagami Fading with EGC Diversity Reception}

We consider in the sequel a $N$-branch EGC receiver operating over Nakagami fadings. The PDF of the Nakagami envelope $R_{i}, i=1,2, \ldots, N$, is

$$
f_{R_{i}}(r)=\frac{2 m_{i}^{m_{i}}}{\Gamma\left(m_{i}\right) \Omega_{i}^{m_{i}}} r^{2 m_{i}-1} \exp \left(-\frac{m_{i}}{\Omega_{i}} r^{2}\right), r>0,
$$

where $m_{i}$ and $\Omega_{i} i=1,2, \ldots, N$, denotes respectively the shape and the spread parameters, whereas $\Gamma(\cdot)$ is the Gamma function. The OCs of the EGC diversity technique that are

(a)

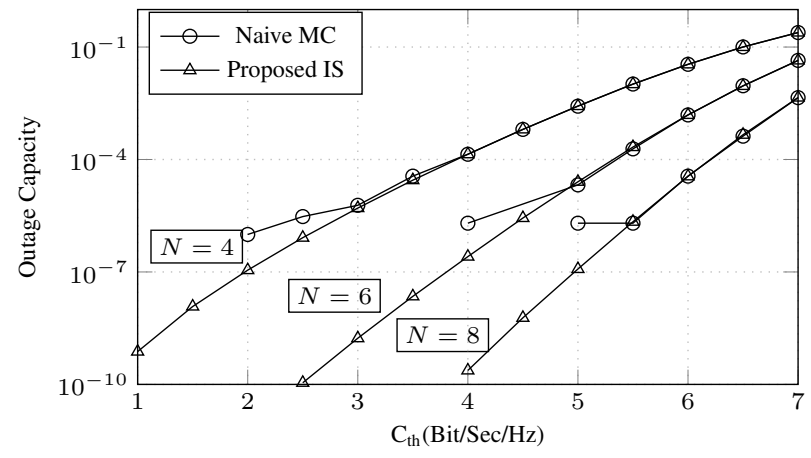

(b)

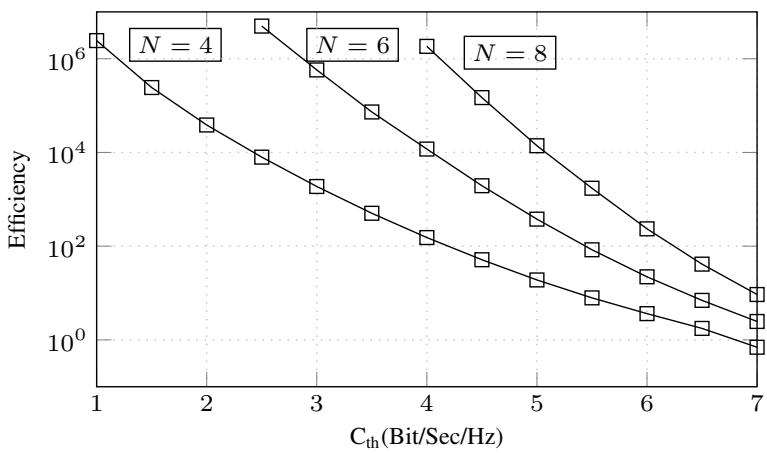

Fig. 3. (a) Outage capacity of $N$-branch EGC diversity receivers over independent Nakagami fading variates with $\Omega_{i}=6, i=1,2, \ldots, 8 . N=4$ : $m=\{0.8,0.8,1.5,1.5\} . N=6: m=\{0.8,0.8,0.8,1.5,1.5,1.5\}$. $N=8: m=\{0.8,0.8,0.8,0.8,1.5,1.5,1.5,1.5\}$. (b) Efficiency $k$ with same parameters. Number of simulation runs: $M=10^{6}$.

estimated by the crude MC simulation and the proposed IS technique are plotted in Fig. 3.a for different number of branches. As in the previous section, we observe that crude MC fails to accurately estimate OC, whereas the proposed IS scheme yields a smoother approximation curve while using the same number of simulation runs. This clearly argues in favor for its efficiency in accurately estimating the OC. Moreover, from Fig. 3.b, it can be easily seen that the IS method achieves a large amount of variance reduction compared to crude MC simulation. For sake of illustration, when $N=4$ and $C_{t h}=1$ $\mathrm{Bit} / \mathrm{Sec} / \mathrm{Hz}$, more than $10^{6} \times M$ simulation runs are needed by crude MC simulations in order to retrieve the accuracy given by the IS scheme with $M$ simulation runs.

\section{CONCLUSION}

We developed a unified hazard rate twisting IS-based simulation approach to efficiently estimate the CDF of the sum of independent arbitrary RVs. This approach serves to accurately estimate the OC with MRC/EGC diversity techniques over independent fading channels. The proposed IS estimator was shown to possess an asymptotic optimality criterion through a minmax selection of the twisting parameter. Based on some selected simulation results, we show that the IS technique achieves a substantial amount of variance reduction compared to crude MC simulations.

\section{REFERENCES}

[1] M.K. Simon and M.-S. Alouini, Digital Communication over Fading Channels, 2nd Edition. New York: Wiley, 2004.

[2] G. L. Stüber, Principles of Mobile Communication, 2nd Edition. Norwell, MA, USA: Kluwer Academic Publishers, 2001.

[3] H. Sandalidis, T. Tsiftsis, G. Karagiannidis, and M. Uysal, "BER Performance of FSO Links over strong atmospheric turbulence channels with pointing errors," IEEE Communications Letters, vol. 12, no. 1, pp. 44-46, Jan. 2008.

[4] N. Sagias and G. Karagiannidis, "Gaussian class multivariate Weibull distributions: theory and applications in fading channels," IEEE Transactions on Information Theory, vol. 51, no. 10, pp. 3608-3619, Oct. 2005.

[5] N. Beaulieu and Q. Xie, "An optimal Lognormal approximation to Lognormal sum distributions," IEEE Transactions on Vehicular Technology, vol. 53, no. 2, pp. 479-489, Mar. 2004.

[6] J. Filho and M. Yacoub, "Simple precise approximations to Weibull sums," IEEE Communications Letters, vol. 10, no. 8, pp. 614-616, Aug. 2006.

[7] D. da Costa, M. Yacoub, and J. Filho, "An improved closed-Form approximation to the sum of arbitrary Nakagami- m variates," IEEE Transactions on Vehicular Technology, vol. 57, no. 6, pp. 3854-3858, Nov. 2008.

[8] K. Peppas, "A simple, accurate approximation to the sum of GammaGamma variates and applications in MIMO free-space optical systems," IEEE Photonics Technology Letters, vol. 23, no. 13, pp. 839-841, Jul. 2011.

[9] N. Beaulieu and F. Rajwani, "Highly accurate simple closed-form approximations to Lognormal sum distributions and densities," IEEE Communications Letters, vol. 8, no. 12, pp. 709-711, Dec. 2004.

[10] J. Hu and N. Beaulieu, "Accurate simple closed-form approximations to Rayleigh sum distributions and densities," IEEE Communications Letters, vol. 9, no. 2, pp. 109-111, Feb. 2005.

[11] S. Chen, H. Nie, and B. Ayers-Glassey, "Lognormal sum approximation with a variant of type IV Pearson distribution," IEEE Communications Letters, vol. 12, no. 9, pp. 630-632, Sept. 2008.

[12] M. Di Renzo, L. Imbriglio, F. Graziosi, and F. Santucci, "Smolyak's algorithm: A simple and accurate framework for the analysis of correlated Log-normal power-sums," IEEE Communications Letters, vol. 13, no. 9 , pp. $673-675$, Sept. 2009.

[13] G. Ropokis, A. Rontogiannis, P. Mathiopoulos, and K. Berberidis, "An exact performance analysis of MRC / OSTBC over generalized fading channels," IEEE Transactions on Communications, vol. 58, no. 9, pp. 2486-2492, Sep. 2010.

[14] S. Juneja and P. Shahabuddin, "Simulating heavy tailed processes using delayed hazard rate twisting," ACM Trans. Model. Comput. Simul., vol. 12 , no. 2 , pp. $94-118$, Apr. 2002.

[15] N. Ben Rached, F. Benkhelifa, A. Kammoun, M.-S. Alouini, and R. Tempone, "Additional results on the hazard rate twisting-based simulation approach," Submitted to IEEE Transactions on Information Theory, 2014. [Online]. Available: http://sri-uq.kaust.edu.sa/Pages/ Page_BenRached_Benkhelifa_Kammoun_Alouini_Tempone_1.aspx

[16] F. Yilmaz and M.-S. Alouini, "A unified MGF-based capacity analysis of diversity combiners over generalized fading channels," IEEE Transactions on Communications, vol. 60, no. 3, pp. 862-875, Mar. 2012.

[17] J. A. Bucklew, Introduction to Rare Event Simulation, ser. Springer series in statistics. New York: Springer, 2004.

[18] J. Sadowsky and J. Bucklew, "On large deviations theory and asymptotically efficient Monte Carlo estimation," IEEE Transactions on Information Theory, vol. 36, no. 3, pp. 579-588, May. 1990.

[19] N. Ben Rached, A. Kammoun, M.-S. Alouini, and R. Tempone, "An improved hazard rate twisting approach for the statistic of the sum of subexponential variates," IEEE Communications Letters, vol. 19, no. 1, pp. 14-17, Jan. 2015.

[20] M. Bagnoli and T. Bergstrom, "Log-concave probability and its applications," Economic Theory, vol. 26, no. 2, pp. 445-469, 2005. 